\title{
Nuevos horizontes para la enseñanza de la fotografía. Retos de la aplicación de proyectos colaborativos y redes multidisciplinares en la enseñanza superior artística.
}

\author{
Ana Martí Testón \\ Universitat Politécnica de Valencia, anmartes@upv.es
}

\begin{abstract}
Photography is an essential artistic and creative discipline that has not acquired the academic acknowledgment it deserves in the university context. In this sense, and struggling to change this perception, and improve its status, from the master's in photography at the Polytechnic University of Valencia, we have worked on different collaborative projects in which the students have been the main axis of the application of new methodologies in which we have created international networks while promoting creativity.
\end{abstract}

Keywords: Photography, art, collaborative, projects, exchanges, International, development, interdisciplinary, higher education.

\footnotetext{
Resumen

La fotografía es una disciplina artística y creativa fundamental que no ha adquirido el reconocimiento académico que merece en el contexto universitario. En este sentido, y luchando por cambiar esta percepción, y mejorar su estatus, desde el Máster en Fotografía de la Universidad Politécnica de Valencia, hemos trabajado en diferentes proyectos colaborativos en los que las alumnas y alumnos han sido el eje principal de la aplicación de nuevas metodologías en las que hemos creado redes internacionales a la vez que fomenamos la creatividad.
}

Palabras clave: Fotografía, arte colaborativo, proyectos, intercambios, Internacional, desarrollo, interdisciplinar, enseñanza superior.

\section{Introducción}

La fotografía es una disciplina que se encuentra a medio camino entre las bellas artes y los medios de comunicación (Chevrier, 2007). Desde su invención en 1839, ha sido utilizada como un medio idóneo para documentar, comprender o interpretar el mundo. Asimismo, ha supuesto una importante aportación a la evolución de las artes y la representación visual, en parte gracias a su capacidad para documentar la realidad, pero también por su habilidad 
para reproducirse, al permitir la amplia circulación y distribución de imágenes. Precisamente en la actualidad, esta capacidad de reproducir la imagen se ha incrementado hasta límites incluso descomunales, debido mayoritariamente a la llegada del Internet y las redes sociales (Fontcuberta, 2016).

De hecho, la fotografía es la disciplina creativa que más gente práctica, y así lo muestran las estadísticas, que ponen de manifiesto como la fotografía, seguida del vídeo o la pintura, es la practica artística más utilizada en el contexto español, como se puede observar en el gráfico de la ilustración 1, extraído de las encuetas realizadas por el Ministerio de Educación respecto a los principales indicadores de prácticas artísticas en 2014-2015 (Subdirección General de Estadística y Estudios, 2015).

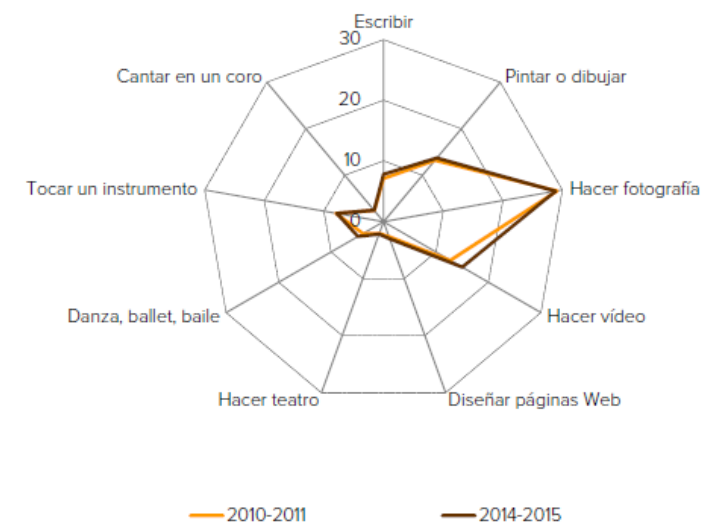

Ilustración 1. Evolución de los principales indicadores de prácticas artísticas. Fuente: Ministerio de Educación, Cultura y Deporte.

Este interés por la fotografía también se hace evidente en la gran cantidad de festivales y concursos que se realizan anualmente en todo el panorama nacional e internacional (Vega, 2017). En los que muchos fotógrafos, aficionados y profesionales, acuden para reunirse con otros colegas y conocer a las figuras importantes del sector. Pero lo cierto es que los usuarios de estos festivales y concursos son en su mayoría personas con un perfil autodidacta (Bourdieu, 2003), puesto que todavía hoy existen pocos lugares oficiales en los que estudiar esta materia. Y pese a que en los últimos años han aparecido distintas escuelas y academias en las que poder estudiar la fotografía (Condés, 2018) en su mayoría se trata de estudios no reglamentados que además presentan un profesorado poco cualificado.

Todavía a principios del siglo XXI, resulta complicada la aceptación de la fotografía en las instituciones educativas superiores, y por ello, desde el Máster en fotografía de la Universidad Politécnica de València llevamos más de 12 años trabajando por cambiar este panorama. Apostando por la enseñanza de la fotografía de calidad, y buscando siempre el máximo reconocimiento en el mundo profesional y artístico.

(cc) EY-NC-ND 2018, Universitat Politècnica de València

Congreso IN-RED (2018) 
El máster nació con el propósito de cubrir algunos de los vacíos que existen todavía hoy en la formación superior de esta disciplina. La mayoría de las universidades públicas a nivel estatal no ofrecen la posibilidad de especializarse en esta materia. Si bien es cierto que se puede estudiar en grados medios y superiores de enseñanzas artísticas, no existe ningún grado específico en ninguna universidad pública en el que estudiar fotografía, y apenas existe reconocimiento dentro de otros grados superiores afines como pueden ser Bellas Artes o Comunicación Audiovisual, carreras en las que apenas se ofrece una asignatura optativa en la que estudiarla (Hernández, 2013). Por ello, la mayoría de los alumnos que vienen a nuestro máster proceden de distintas carreras con perfiles muy variados, lo que supone un importante reto pedagógico a la hora de trabajar con las capacidades técnicas y creativas de las alumnas y alumnos.

Si bien es cierto que la técnica es una parte imprescindible en la enseñanza de la fotografía, debemos señalar que ésta por sí sola no es capaz de transmitir significado a las obras. Por lo tanto, en la docencia de este tipo de disciplina, se deben desarrollar al mismo tiempo las destrezas técnicas y las capacidades creativas (Bridges, 2013). Pues no se trata sólo de hacer una buena fotografía, sino de analizar y explicar por qué queremos hacerla.

Desde nuestro grupo de trabajo creemos importante desarrollar metodologías que fomenten la creatividad, y no se queden ancladas en la obsesión por perfeccionar la técnica. Pero la posibilidad de desarrollar competencias creativas en los alumnos de postgrado que provienen, como ya hemos indicado, de diferentes perfiles, resulta realmente complicado, y supone un reto para los docentes de esta materia. De hecho, fomentar la creatividad y las ideas originales resulta bastante más complejo que el reto de enseñar la técnica fotográfica, pues normalmente ésta requiere un mayor nivel intelectual (Sontag, 2010). Asimismo, la parte técnica es más fácilmente evaluable y objetivable que la parte creativa. Con demasiada frecuencia, y especialmente los estudiantes más jóvenes, interpretan erróneamente el éxito creativo de las obras basándose en la técnica, mientras que el problema creativo reside fuera de ésta, y se debe trabajar y mejorar independientemente de las herramientas que se utilicen (Bridges, 2013), como ocurre en el desarrollo de otras enseñanzas artísticas.

\section{Objetivos}

Uno de los principales objetivos al que nos enfrentamos es experimentar nuevos modos de enseñanza-aprendizaje de la fotografía en el marco superior basados en la experimentación práctica en las que poder explotar las capacidades creativas de los alumnos en cuanto al desarrollo de ideas innovadoras.

Con la intención de fomentar la creatividad en los alumnos y alumnas que provienen de distintos ámbitos, con distintas capacidades, hemos organizado diferentes actividades colaborativas en las que han podido desarrollar sus intereses y descubrir nuevas posibilidades expresivas en el ámbito del arte y el comisariado de exposiciones.

Otro objetivo importante de este tipo de actividades es ampliar los horizontes culturales de los alumnos, e intercambiar experiencias con otros masters de distintos países con los que 
hemos colaborado con el fin de mejorar la calidad de la enseñanza superior en fotografía. Especialmente en lo que se refiere a la capacidad de comprender nuevas posibilidades para aprender esta materia y relacionarla con otras disciplinas artísticas.

Asimismo, queremos ir un paso más allá en la enseñanza de la fotografía como una disciplina artística y alejarnos de la obsesión por la mejora de la técnica, para centrarnos en la posibilidad de crear proyectos comunes y colaborativos que se aproximen a la realidad a la que se enfrentan los artistas contemporáneos.

Uno de los objetivos de la colaboración con otros másteres es la difusión del trabajo de los alumnos y ex alumnos de las universidades que forman la red. Asimismo, hemos apostado por avanzar en la innovación pedagógica en fotografía, propiciando el intercambio de alumnos y profesores, y la producción de proyectos colaborativos.

El objetivo de estas experiencias es buscar nuevas maneras de ampliar los limites convencionales expositivos de la fotografía y mejorar la comunicación de estas pensando en el nuevo público contemporáneo y heterogéneo. Otro objetivo es llevar a los alumnos fuera del ámbito académico para que se relacionen con otros entornos con los que deberán trabajar una vez terminen sus estudios.

\section{Desarrollo de la innovación}

Con la idea de mejorar la creatividad de los alumnos y las alumnas y dar prioridad a sus intereses, nos hemos basado en distintas metodologías contemporáneas que hemos puesto en práctica en el desarrollo de varias experiencias innovadoras. Una de ellas es la metodologia del Design Thinking, o pensamiento del diseño, una metodologia que proviene del mundo de los creativos y diseñadores, y cuenta con una parte de aplicación práctica importante para el desarrollo y materialización de las ideas creativas y originales, que consideramos es ideal en el desarrollo de las capacidades artísticas. Para ello, se propuso realizar proyectos colaborativos entre distintos grupos de alumnos en los que utilizaríamos esta metodología para aprender de los errores y reconstruir las ideas en función de éstos. Los alumnos son los encargados de desarrollar su propia experiencia y deben aprender mediante la práctica colaborativa de organizar una exposición, o un evento, que se debía desarrollar de una manera poco habitual en un espacio no convencional.

La idea que propusimos era escoger un tema genérico que se trabajó por los distintos grupos. El curso pasado el tema escogido fué el "cambio". Las alumnas y los alumnos del máster debían desarrollar distintos proyectos expositivos en los que trabajaron con la fotografía, el video y las artes visuales, en entornos poco habituales, en base a la idea del cambio. En función de la aproximación al tema que habían escogido, debían realizar una primera fase de sensibilización para empezar a diseñar y presentar sus propuestas expositivas. Una vez analizados los distintos puntos de vista de los grupos, y las ideas propuestas por cada uno, se pasó a la definición concreta de la solución escogida y su desarrollo, para posteriormente materializarlo y evaluarlo según los resultados, y finalmente realizar los cambios pertinentes y determinar la presentación final. Un ejemplo realizado por un grupo de alumnos del curso pasado fue la realización una instalación artística y un

(cc) BY-NC-ND 2018, Universitat Politècnica de València

Congreso IN-RED (2018) 
concierto, que tuvo lugar en un solar del barrio del Cabañal de Valencia, hablando de las personas que habían habitado este espacio antes de ser derruido, y utilizaron la fotografía y la imagen como un nexo para explicar su evolución y desolación. Otro grupo decidió realizar la publicación de un fanzine para el que contaron con la colaboración de distintos

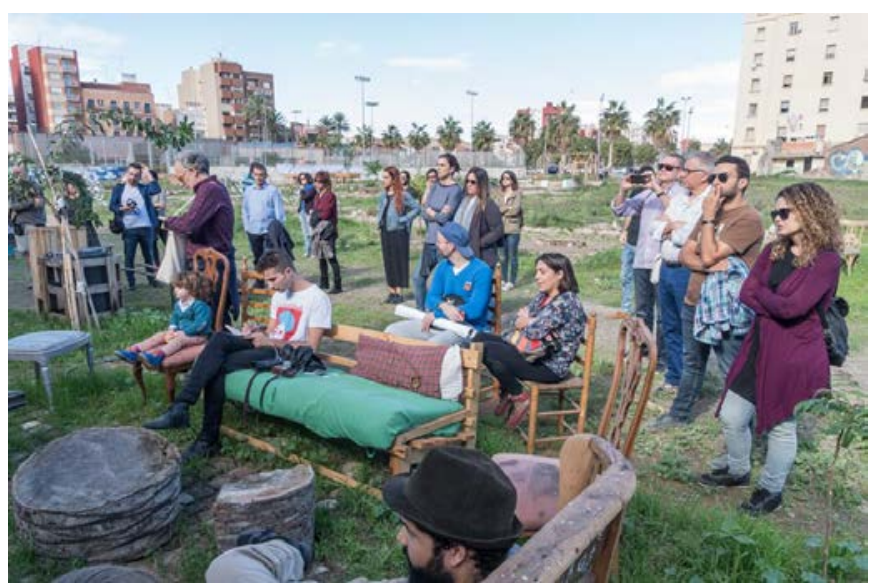

Ilustración 2. Ejemplo de intervención en un solar del Cabañal de Valencia.

fotógrafos y escritores o críticos que trataron el tema del cambio.

Utilizando esta misma metodología, propusimos a los alumnos que se encargaran de comisariar una galería en el centro de la ciudad de Valencia, en la Fundación La Posta, en la que debían ponerse de acuerdo para ver qué tipo de contenidos querían exponer y como podían dinamizar el local para fomentar el interés de distintos perfiles de visitantes. La mayoría de los alumnos apenas contaban con experiencia en este campo, y tuvieron que aprender a marchas forzadas mediante la práctica y experimentando distintos errores de los que posteriormente aprendieron. El grupo de alumnas y alumnos que se encargaron del espacio realizaron un borrador de propuestas que posteriormente evaluamos los docentes del máster para terminar de perfilar la propuesta expositiva, y en base a ello, comenzar a materializar las ideas. Cada alumno debía encargarse de una tarea, entre las que se encontraba el diseño de los carteles y la publicidad, la comunicación de los eventos y las exposiciones, la financiación, las actividades relacionadas, los contactos, etc.

Otro proyecto diferente que hemos realizado en los últimos años con la idea de ampliar las miras de los alumnos y mejorar su capacidad crítica, es la colaboración con otros alumnos de otros másteres internacionales. En esta experiencia pusimos en práctica la metodología ya bien conocida de las clases invertidas "flipped Classroom", en las que se fomenta que en el aula se realicen las cuestiones prácticas y se deja para casa, o para que cada alumno desarrolle a su ritmo, la parte de investigación teórica. En este tipo de metodología, contraria a las clases magistrales tradicionales, el alumno adquiere un rol más activo, pues ellos mismos eligen el momento y modo en que realizan el aprendizaje, motivando una vez más el pensamiento crítico al enfrentarse a distintos problemas complejos para los que ya cuentan con la ayuda del profesor o docente dentro del aula. Para ello, se han aprovechado además las oportunidades que nos brindan los medios digitales y de comunicación, que se 
han utilizado para que las alumnas y alumnos se comunicaran con otros estudiantes de otros másteres internacionales.

El principal objetivo de este proyecto era crear una red de universidades que nos ayudase a mejorar la calidad de la enseñanza y mismo tiempo que nos posibilitara la capacidad de ampliar los horizontes pedagógicos con nuevas experiencias que nos van enriqueciendo. Por ello, decidimos huir de la enseñanza al estilo "tradicional” de seminarios y clases teóricas, para apostar por una enseñanza activa en la que buscamos desarrollar nuevas experiencias enriquecidas gracias a la colaboración, investigación y desarrollo de los proyectos conjuntos de los alumnos (Ivern Magaña, 2009).

Gracias a la experiencia que habíamos acumulado en los últimos años, al colaborar con distintas universidades en proyectos europeos de investigación, en el año 2016 decidimos empezar a desarrollar una red europea de estudios superiores en fotografía: ENMAP: European Network of Máster Studies in Photography ${ }^{1}$. Una red formada por nueve universidades europeas con estudios de postgrado en fotografía entre las que se encuentran la universidad de Aalto en Finlandia, la Ecole de Recherche Graphique de Bruselas, Falmouth University, Leiden University, London College of Communication, Plymouth University o la Universidad de Westminsiter.

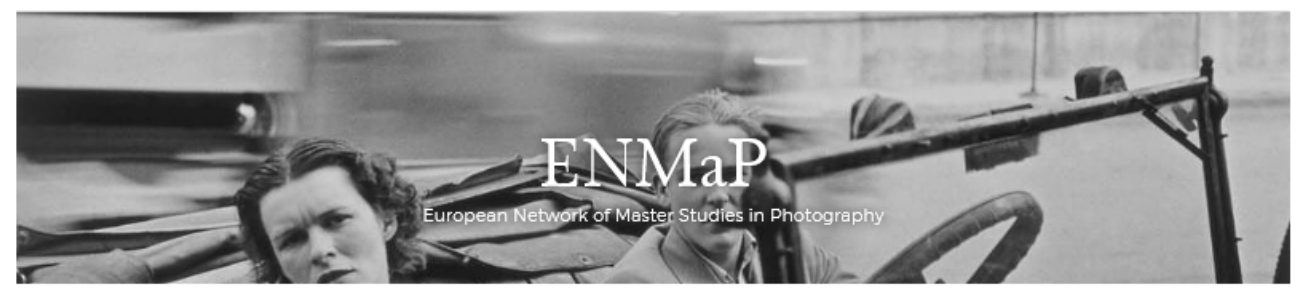

Ilustración 3. Cabecera de la web de la asociación ENMAP

Todas las universidades del consorcio cuentan con importantes programas docentes y un reconocido prestigio internacional en el ámbito de las enseñanzas artísticas y especialmente en el campo de la fotografía. En una primera reunión los socios presentamos los distintos programas educativos y lo primero que nos llamó la atención eran las grandes diferencias que existen en los programas de cada universidad.

Existen muchas diferencias en el estatus de la fotografía como disciplina artística en el ámbito universitario, entre los distintos países europeos, y esto se evidencia en los planes de estudios con distintas formas de enseñar y de enfocar el proceso de enseñanza aprendizaje. A partir de la posibilidad de compartir estas experiencias pudimos aprender de las prácticas de las otras universidades. Por ejemplo, el programa del Máster en fotografía de la UPV está organizado con bastantes horas de carga docente de profesores invitados que vienen al máster a hacer máster class, clases magistrales. El programa se divide en dos grandes bloques, por un lado, la teoría y por otro lado la práctica; El máster de Leiden es un máster

${ }^{1} \underline{\text { http://gr3.be/enmap/ }}$

(cc) EY-NC-ND 2018, Universitat Politècnica de València

Congreso IN-RED (2018) 
teórico más enfocado hacia la hacia el comisariado de exposiciones y la crítica; La situación de las universidades inglesas es totalmente diferente, puesto que los socios ingleses cuentan con alumnos que normalmente han estudiado un grado fotografía, cosa que en España no es posible de momento (ni en otros países europeos). Los programas de las universidades inglesas que participan en el consorcio son bastante parecidos entre ellos; normalmente mezclan la teoría con la práctica y tienen muy pocas horas de carga docente, dejando mucha libertad a los alumnos y el desarrollo de sus propios proyectos artísticos. En el caso del máster de la Escuela de Arte de Bruselas, se trata unos estudios mucho más integrados en las bellas artes, y no son tan cerrados a la fotografía, con procesos creativos multidisciplinares como pueden ser la performance o el vídeo. Rápidamente nos dimos cuenta de que la mayoría de los programas se podían complementar unos a otros, y podríamos compartir experiencias, así como profesores y alumnos, y empezamos a trabajar en las distintas líneas de analizar de qué manera podríamos sacar provecho.

Se propuso la realización de una exposición conjunta en la que se mostrasen los trabajos de los alumnos de los distintos másteres, pero quisimos que esta exposición fuera diseñada y organizada por los propios alumnos y las alumnas del máster de Valencia, para que fueran ellos, mediante el proceso del aprendizaje por proyectos colaborativos, quienes nos fueran pidiendo a los profesores los recursos que necesitan para desarrollar sus ideas.

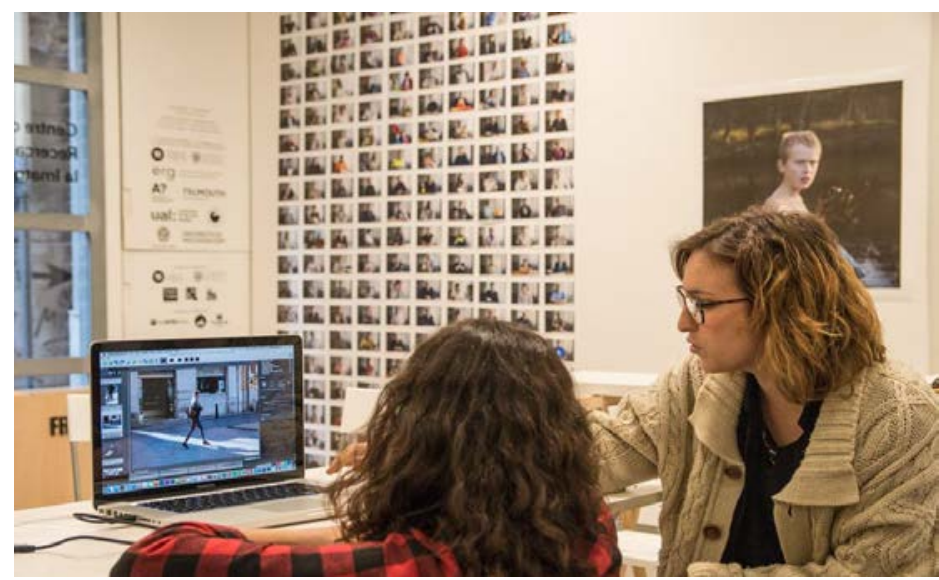

Ilustración 4. Unas alumnas debaten sobre cómo realizar un proyecto colaborativo.

Con el objetivo de escoger un tema que englobase los trabajos expuestos de los alumnos y alumnas de los distintos másteres, los alumnos de la UPV se tuvieron que poner en contacto con los compañeros de otros países y preguntarles por los detalles de los proyectos que habían desarrollado. Como un medio espontáneo para establecer estas comunicaciones se popularizó el uso de las redes sociales, y especialmente Facebook, para comunicarse entre ellos. Como resultado de estas comunicaciones, se crearon debates y foros en los que intercambiaron opiniones y materiales hasta escoger el tema que los podía englobar: "NeXoS". 


\section{Resultados}

Después de la realización de los distintos proyectos efectuamos sesiones de evaluación con los alumnos implicados con la intención de analizar los puntos positivos y las limitaciones con las que se habían encontrado.

Una de las primeras conclusiones que empezamos a recoger de estas experiencias es que cuanto más radical era la propuesta que les planteábamos, más efectivo era el proceso de aprendizaje. El hecho de proponer la realización de una exposición con un formato innovador fomentó la creación de ideas originales que surgieron a partir de las experiencias de cada uno de los alumnos, ya que aportaron los recursos de los que disponían. De este modo, aprendieron bastante más de lo que podían haber aprendido con una clase magistral de comisariado de exposiciones. Asimismo, hemos podido comprobar cómo estas experiencias colectivas han supuesto una lanzadera para futuros proyectos profesionales de los alumnos, que al terminar el máster han optado por desarrollar proyectos innovadores en el campo profesional de la fotografía, por ejemplo, con la promoción de publicaciones o el

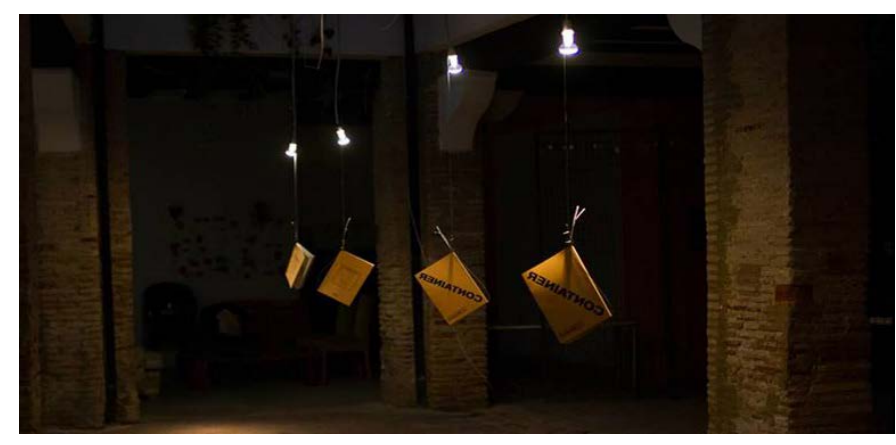

Ilustración 5. Muestra de una de las exposiciones de los alumnos en la que mostraban una publicación conjunta

comisariado de exposiciones.

En los últimos dos años hemos realizado diversas acciones que han resultado de gran ayuda para la formación artística de los alumnos del máster. Por un lado, como consecuencia de la creación de la red de másteres internacionales, realizamos intercambios de profesorado e invitamos a profesores de otros másteres a impartir seminarios en el nuestro y viceversa. De este modo, completábamos los programas curriculares con otras asignaturas que podían complementar a las ya impartidas y aprovechamos para mantener el contacto con los diferentes profesores y alumnos de otras universidades. De hecho, esta oportunidad ha posibilitado la creación de otras experiencias diferentes, como puede ser invitar a algunos alumnos a participar en exposiciones conjuntas realizadas en otros países, o la posibilidad de hacer encuentros en verano en festivales de fotografía.

Por otro lado, realizamos distintas clases online en las que utilizamos los recursos de streaming de que dispone la Universitat Politécnica de Valencia para compartir las experiencias con los otros másteres. Además, de la ya comentada exposición en las que se

(cc) EY-NC-ND 2018, Universitat Politècnica de València

Congreso IN-RED (2018) 
seleccionaron los trabajos de los alumnos que terminaron ese año los estudios. De hecho, fueron los propios alumnos quienes seleccionaron los artistas que podrían exponer siguiendo una idea o un tema expositivo que había sido escogido por ellos. Se determinó que, en el futuro, cada año, una universidad diferente se encargaría de organizar la exposición en su país con la posibilidad de que itinere a otros países de los otros másteres. Como resultado de esta primera experiencia realizada por los alumnos de Valencia, se decidió que los alumnos de la universidad de Leiden se encargan de escribir los textos a modo de crítica artística sobre los trabajos expuestos, puesto que provienen de un máster fundamentalmente teórico. El resultado fue realmente interesante, e incluso se logró que la exposición formase parte del festival FotOn sobre fotografía y fotoeriodismo que se desarrolla en la ciudad de Valencia.

Esta primera experiencia piloto fue muy positiva, y los alumnos han declarado sentirse muy satisfechos con ella. De hecho, al comparar la experiencia y los resultados obtenidos con alumnos de ediciones anteriores, nos hemos percatado de que el nivel de los proyectos personales y artísticos de los alumnos también había mejorado, probablemente enriquecidos por todas estas actividades paralelas realizadas en los distintos proyectos.

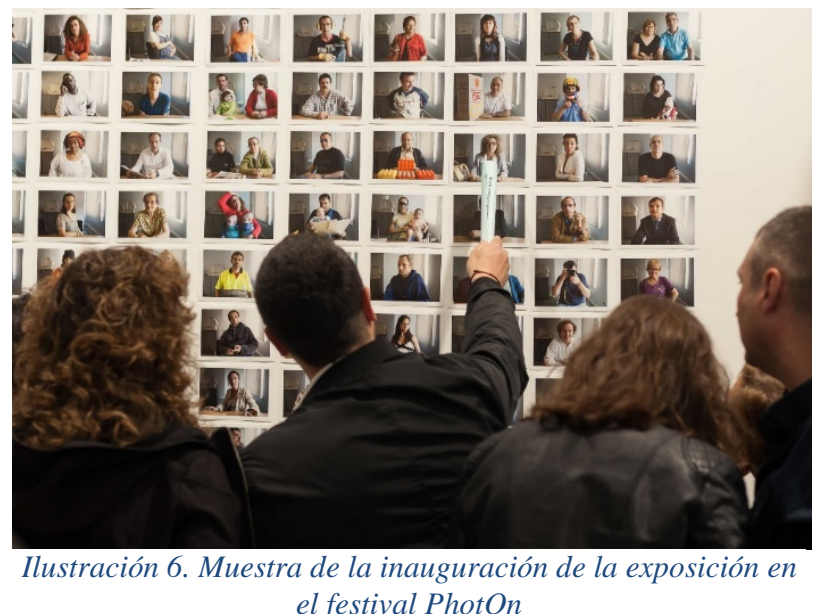

\section{Conclusiones}

Después de más de un siglo desde que naciera la fotografía, hemos podido comprobar como esta disciplina se ha popularizado en la sociedad. Pero lamentablemente en lo que se refiere a innovaciones pedagógicas en el campo de la educación artística, apenas ha evolucionado. El modo que prevalece por excelencia es el de clases magistrales y exposiciones de trabajos de los alumnos en espacios convencionales como museos o galerías (Jackson, 2007).

Pero el uso de tecnologías educativas emergentes ha supuesto un reto que muchos docentes están aprovechando. En concreto, nosotros, las hemos aprovechado para el desarrollo de proyectos colaborativos como los que hemos comentado en este artículo, y gracias a ellas, hemos podido poner en práctica la metodologia del aula invertida ya comentada. Las tecnologías digitales nos han permitido ayudar a los alumnos a acceder a los contenidos 
cuando ellos lo deseaban, fuera y dentro del aula, permitiendo el acceso continuo tanto dese ordenadores como dispositivos móviles. De este modo, los estudiantes se han convertido en los responsables de gestionar su aprendizaje, y así lo ha demostrado su evaluación positiva de la experiencia. Asimismo, han declarado que las temáticas de los proyectos se han ajustado mejor a las expectativas personales de aprendizaje de cada uno de ellos.

Por otro lado, estas tecnologías han servido de puente entre los alumnos y profesores de nuestro máster con otros alumnos y profesores de otros másteres internacionales. Gracias a internet y la posibilidad de comunicarnos y conocernos mediante las redes sociales, el correo electrónico y las plataformas como Polimedia, hemos reducido las distancias y hemos colaborado con otros profesionales y estudiantes que se encuentran lejos, propiciando el interés e incluso la posibilidad de conocerse en persona.

Con la intención de realizar una evaluación más objetiva de las capacidades artísticas de los alumnos y las alumnas, determinamos que cada grupo se debía de encargar de evaluar las propuestas de los otros compañeros. $\mathrm{Y}$ a pesar de que esta idea en un primer momento resultó chocante para algunos de ellos, posteriormente reconocieron que el hecho de evaluar el trabajo de otros les había servido para realizar una reflexión autocrítica con su propio trabajo. Posteriormente, los profesores y profesoras del máster realizamos también nuestra valoración de las actividades y su evaluación de acuerdo con unos valores ya indicados con anterioridad.

Por otro lado, queremos comentar como nos sorprendió que no todos los alumnos se mostraran igual de contentos con la idea de trabajar en grupos, y en algunos grupos, de hecho, surgieron roces y problemas que afectaron al normal desarrollo de las actividades. Como docentes, estamos sorprendidos con la poca capacidad de los alumnos de trabajar en equipo, y la necesidad de muchos de ellos de mostrar su liderazgo o los problemas surgidos por el exceso de ego, - que es bastante normal en los alumnos de bellas artes, especialmente en los primeros cursos, pero no es tan común a nivel de máster-. De hecho, nos sorprende que, en lugar de encontrarnos limitaciones a la hora de aplicar metodologías innovadoras en espacios expositivos no convencionales, los problemas surgieran de algo tan básico como es el trabajo en grupo. Efectivamente, creemos que esto mismo supondrá una barrera para estos alumnos que se tendrán que enfrentar al terreno profesional, dónde su trabajo será continuamente cuestionado, y cuando en muchas ocasiones se tendrán que desenvolver al trabajar en equipo.

No obstante, en todo momento hemos intentado fomentar las capacidades creativas de los alumnos que acuden a estudiar un máster en fotografía para completar su formación. Muchos de ellos llegan con grandes esperanzas y expectativas que no siempre se cumplen, pero desde nuestra postura, como docentes, debemos intentar facilitarlo en la medida que sea posible. De hecho, los primeros beneficiados de todos los proyectos que realizamos son los alumnos, y no los profesores, pues para los profesores suele suponer un sobreesfuerzo, que no siempre es recompensado. Pero, aunque estamos más cansados, estamos encantados de poder tener la oportunidad de hacerlo, pues, aunque realizamos estas actividades paralelamente al currículum oficial, esperamos que en ediciones posteriores este tipo de

(cc) EY-NC-ND 2018, Universitat Politècnica de València

Congreso IN-RED (2018) 
proyectos puedan tener un mayor protagonismo en las guías docentes, y que los alumnos y las alumnas puedan valorar y aprovechar el aprendizaje adquirido.

Estamos convencidos de que cuanto más radicalizamos las metodologías de aprendizaje experimental mediante el desarrollo de proyectos colaborativos, como los que hemos expuesto, basados en el uso del pensamiento del diseño, el pensamiento crítico y la propuesta del aula invertida, podemos mejorar cualitativamente las capacidades creativas de las alumnas y los alumnos.

\section{Referencias}

Bourdieu, P. (2003). Un arte medio. Barcelona: Gustavo Gili.

Bridges, M. (2013). Creativity Education. En G. Rand, \& R. D. Zakia, Teaching Photography. London: Elseiver. Obtenido de Teaching Photography : https://www.safaribooksonline.com/library/view/teachingphotography/9780240807676/013_9781136105890_chapter5.html

Chevrier, J.-F. (2007). La fotografía entre las bellas artes y los medios de comunicación. Barcelona: Gustavo Gili.

Condés, O. (4 de mayo de 2018). ¿Cuál es el mejor método para estudiar fotografía? Obtenido de Xataka Foto: https://www.xatakafoto.com/trucos-y-consejos/cual-esel-mejor-metodo-para-estudiar-fotografia

Fontcuberta, J. (2016). La furia de las imágenes. Notas sobre la postfotografía. Barcelona: Galaxia Gutenberg.

Hernández, A. L. (16 de diciembre de 2013). ¿Dónde estudiar fotografía? Recuperado el 18 de marzo de 2018, de Foto Blogger.

Ivern Magaña, J. (Junio de 2009). Por una nueva metodología en los estudios superiores de las artes. Recuperado el 20 de marzo de 2018, de http://www.raco.cat/index.php/Observar/article/viewFile/179280/231740

Jackson, F. (2007). Teaching Photography as Art: A Short Critical History. Smithsonian American Art Journal, 21(3), 19-24.

Sontag, S. (2010). Sobre la fotografía. Barcelona: Debolsillo.

Subdirección General de Estadística y Estudios. (2015). Encuesta de hábitos y prácticas culturales en España 2014-2015. Madrid: Ministerio de Educación, Cultura y Deporte.

Vega, C. (2017). Fotografía en España (1839-2015). Ediciones Cátedra. 